\title{
A structural view of the conserved domain of rice stress-responsive NAC1
}

\author{
Qingfeng Chen ${ }^{1}$, Quan Wang ${ }^{3}$, Lizhong Xiong ${ }^{1 凶}$, Zhiyong Lou ${ }^{2 凶}$ \\ 1 National Key Laboratory of Crop Genetic Improvement, National Center of Plant Gene Research (Wuhan), Huazhong \\ Agricultural University, Wuhan 430070, China \\ 2 Structural Biology Laboratory, Tsinghua University, Beijing 100084, China \\ ${ }^{3}$ College of Life Sciences and Tianjin State Laboratory of Protein Science, Nankai University, Tianjin 300071, China \\ $\bowtie$ Correspondence: lizhongx@mail.hzau.edu.cn (L. Xiong), louzy@xtal.tsinghua.edu.cn (Z. Lou) \\ Received January 1, 2011 Accepted January 20, 2011
}

\begin{abstract}
The importance of NAC (named as NAM, ATAF1, 2, and CUC2) proteins in plant development, transcription regulation and regulatory pathways involving proteinprotein interactions has been increasingly recognized. We report here the high resolution crystal structure of SNAC1 (stress-responsive NAC) NAC domain at $2.5 \AA$. Although the structure of the SNAC1 NAC domain shares a structural similarity with the reported structure of the ANAC NAC1 domain, some key features, especially relating to two loop regions which potentially take the responsibility for DNA-binding, distinguish the SNAC1 NAC domain from other reported NAC structures. Moreover, the dimerization of the SNAC1 NAC domain is demonstrated by both soluble and crystalline conditions, suggesting this dimeric state should be conserved in this type of NAC family. Additionally, we discuss the possible NAC-DNA binding model according to the structure and reported biological evidences.
\end{abstract}

KEYWORDS stress-responsive NAC 1, NAC family, DNA binding, rice, crystal structure

\section{INTRODUCTION}

Transcription factors are important components in transcriptional regulation and are involved in virtually every aspect of biologic activities in eukaryotes. More than 50 known transcription families have been revealed through computational analysis of the genome of Arabidopsis and rice (Riechmann et al., 2000; Xiong et al., 2005).

NAC (named as NAM, ATAF1, 2, and CUC2) transcription factors, which are unique to plants, constitute one of the largest plant transcription factor families, of which 105, 138 and 163 putative NAC members have been identified in Arabidopsis, rice and Populus genomes respectively (Ooka et al., 2003; Fang et al., 2008; Hu et al., 2010). Among the three members, after which this family is named, NAM (No Apical Meristem) from petunia is important for shoot apical meristem formation (Souer et al., 1996), CUC2 (cup-shaped cotyledon) is involved in controlling the formation of boundary cells of the meristem (Aida et al., 1997), and ATAF1, 2 show dual function in biotic and abiotic stress responses (Collinge and Boller, 2001; Delessert et al., 2005; Jensen et al., 2007; Lu et al., 2007; Jensen et al., 2008; Wang et al., 2009; Wu et al., 2009).

With the large number of family members in plant genomes, function redundancy is inevitable for NAC proteins. Besides NAM, CUC1 and CUC2, CUC3, NAP (Sablowski and Meyerowitz, 1998) and CUP (Weir et al., 2004) from snapdragon also participate in the establishment of organ boundary and participates in the formation of shoot meristem. Participation of NAC proteins in other developmental processes has also been reported. NAC1 was found to act downstream of TIR1 and activate the expression of two auxinresponsive genes DBP and AIR3, therefore promoting lateral root development (Xie et al., 2000). VND6 and VND7 are involved in xylem formation (Kubo et al., 2005; Demura and Fukuda, 2007; Du and Groover, 2010), while ANAC012 functions in xylary fiber development (Ko et al., 2007). Several NAC members including SND1, NST1 and NST3 were found to regulate secondary wall synthesis (Zhong et al., 2006; Mitsuda et al., 2007; Zhong et al., 2007a, b; McCarthy et al., 2009). Notably, a few members have been reported to participate in multiple processes, which might be a general rule for most NAC proteins. For example, a member from wheat regulates senescence and at the same time controls grain size, zinc and ion content (Uauy et al., 2006) and a 
membrane-associated NAC protein from Arabidopsis regulates cell division as well as salt-responsive flowering (Kim et al., 2006; Kim et al., 2007). In addition to ATAF1, 2, a number of NAC proteins were shown to play roles in biotic and abiotic responses. OsNAC6, an ATAF homolog in rice, is rapidly and strongly induced by cold, salt, drought, abscisic acid, jasmonic acid and wounding, indicating its role in both biotic and abiotic responses (Ohnishi et al., 2005; Nakashima et al., 2007). Recently, OsNAC5, like OsNAC6, was characterized as a transcriptional activator that enhances stress tolerance by upregulating the expression of stress-inducible rice genes (Sperotto et al., 2009; Takasaki et al., 2010). Using mRNA differential display, StNAC, an ATAF homolog in potato, was found to be induced early after wounding (Collinge and Boller, 2001). ANAC019, ANAC055, and ANAC072, which also belong to ATAF subfamily, show enhanced drought tolerance when overexpressed (Tran et al., 2004). There are eight NAC genes that have been found to be differentially regulated in Brassica napus leaves subjected to mechanical wounding, flea beetle feeding or cold temperatures (Hegedus et al., 2003). Most strikingly, overexpression of stress responsive gene SNAC1 (STRESSRESPONSIVE NAC 1) from rice significantly enhances drought resistance while showing no phenotypic changes or yield penalty and shows great promise for genetic improvement of stress tolerance in rice (Hu et al., 2006).

Although NAC family transcription factors play key roles in plant, limited knowledge of their detailed structures or DNA binding mechanism is known and only one crystal structure of NAC family protein has been previously reported (Ernst et al., 2004). To gain a better understanding of the structural details of NAC family transcription factors, here we describe the crystal structure of the SNAC1 NAC domain and show that the overall architecture adopts a conserved NAC family fold. Key features, however, distinguish the SNAC1 NAC domain from reported ANAC structures, including two loop regions which could be crucial for DNA binding. To gain better insight into the DNA binding of SNAC1 NAC domain, we propose a model of SNAC1 NAC domain in complex with the $35 \mathrm{~S}$ promoter that reveals biochemically well-defined sites that help to account for strict substrate-recognition motifs.

\section{RESULTS AND DISCUSSION}

\section{Monomer folding of SNAC1 NAC domain}

The crystal structure of the conserved NAC domain of stressresponsive NAC1 (SNAC1) (residues Met1-Lys174) was determined by the molecular replacement (MR) method using the crystal structure of the NAC domain of ANAC (PDB code: 1UT4) (Ernst et al., 2004) as the initial searching model. The final structure was refined to $2.5 \AA$ resolution with a final $R_{\text {work }}$ value of $23.1 \%\left(R_{\text {free }}=26.7 \%\right)$. The crystal belongs to the space group $P 4_{1} 2_{1} 2$, and there is one molecule in an asymmetric unit with a Matthews coefficient of $2.6 \AA^{3} / \mathrm{Da}$ (corresponding to $43 \%$ solvent content) (Matthews, 1968).

The SNAC1 NAC domain monomer consists of a central semi- $\beta$-barrel formed by seven twisted anti-parallel $\beta$-strands with three $\alpha$-helices on one side and the open side respectively (Fig. 1A). The remaining part of the structure primarily consists of loop regions with several parts. Loop regions Gly144-Gly149 and Lys180-Asn183 were not observed due to lack of electron density, suggesting their high flexibility and indicating their conformation is highly flexible. Notably, one of those loop regions, e.g. Gly144-Gly149, has the most distinctive difference between the SNAC1 NAC domain and ANAC NAC domain (Fig. 2A). As SNAC1 NAC has a longer amino acid sequence in this region, we speculate that this region could be crucial for the biologic role of the SNAC1 NAC domain, which might be different from the ANAC NAC domain. Nonetheless, according to the high amino acid sequence similarity $(60 \%)$ with ANAC, the central part of the SNAC1 NAC domain unexpectedly shares a high topological similarity with the structure of ANAC (PDB code: 1UT4) (Ernst et al., 2004) as well. However, distinct structural differences are observed between these two structures. First, the $\mathrm{N}$-terminal extended residues in the structure of ANAC NAC domain are involved in contacts with symmetric molecule and thus have relative good agreement (Ernst et al., 2004). In contrast, although there are several residues that remain in the $\mathrm{N}$-terminus of the SNAC1 NAC domain caused by the expression vector, all of these residues are totally unobserved in the crystal structure of SNAC1 NAC domain suggesting a high flexibility. Second, although the residues of the loop regions between $\beta 1-\beta 2$ and $\beta 6-\beta 7$ in both SNAC1 and ANAC NAC domains are missing due to lack of electron density and the number of unobserved residues in the ANAC NAC domain is obviously more than that of SNAC1 NAC domain. As the two regions do not participate in crystal packing in both structures, it reveals that the flexibility and conformation of these two regions could be different. Considering the amino acid sequence disalignment of these regions, we would like to propose that this difference could be related to their different biologic functions. Although there are several other differences that could be observed in the crystal structure of SNAC1 and the ANAC NAC domains, the slight differences could be caused by the difference in the amino acid sequences and seems not to be related to their biologic function.

\section{Dimeric assembly of SNAC1 NAC domain}

Although there is only one molecule of SNAC1 NAC domain in each asymmetric unit in this crystal form, the results of gel filtration and analytical ultracentrifugation show that the SNAC1 NAC domain exists as a dimer in solution. Subsequently, the results of ethylene glycol disuccinate di $(\mathrm{N}$ succinimidyl) ester (EGS) cross-linking confirmed this observation and demonstrated that such a dimeric architecture does not dissociate, even at very high salt concentrations 
A


B
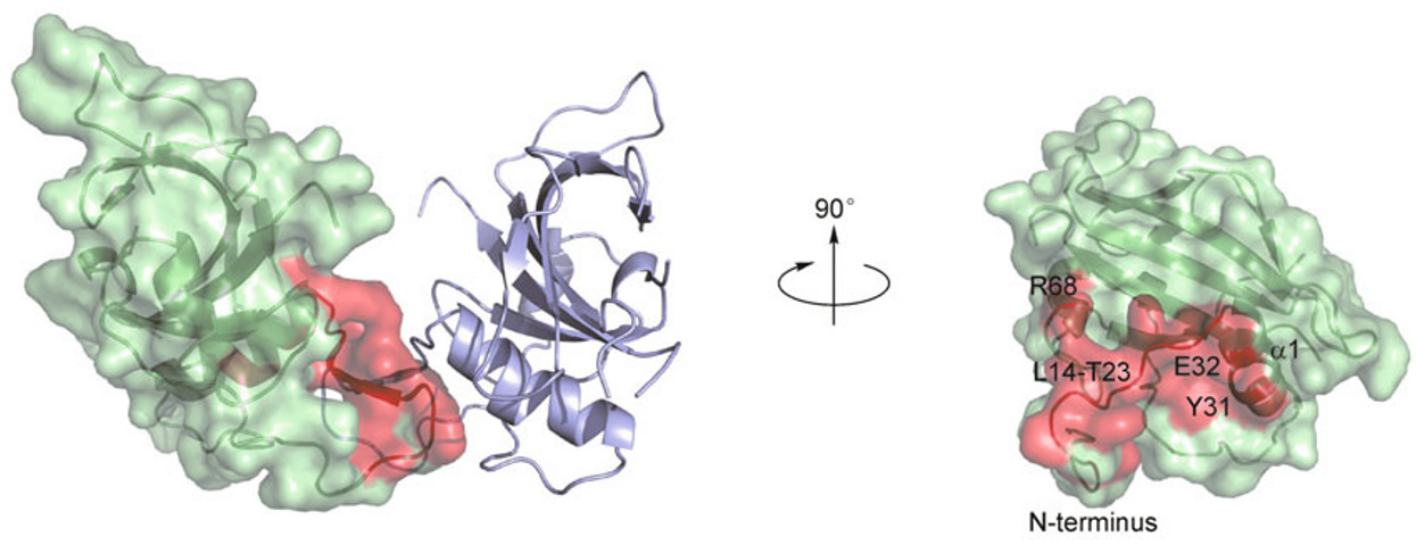

Figure 1. Crystal structure of SNAC1 NAC domain. (A) The overall structure of the SNAC1 NAC domain monomer. The monomer structure of the SNAC1 NAC domain is colored by secondary structure with helices and strands colored pink and dark green respectively. (B) The structure of homodimeric SNAC1 NAC domain. The molecules are colored green and blue respectively, while the interface between two monomers is colored red. The residues, which are crucial for intermolecular interaction (distance less than $3.6 \AA$ ), are labeled.

$(5 \mathrm{M} \mathrm{NaCl})$. These results indicate that the dimeric form should form the functional unit of the SNAC1 NAC domain, which is consistent with the previously reported results on the ANAC NAC domain (Ernst et al., 2004) and our knowledge that the dimerization of DNA binding domains is common and can function in modulating the DNA binding specificity (Müller, 2001). In the crystal structure, two molecules of the SNAC1 NAC domain, which are related by a crystallographic twofold axis, form a butterfly shaped homodimer (Fig. 1B). According to the space group of $P 4_{1} 2_{1} 2$, two types of homodimers could be generated via two vertical crystallographic twofold axises. Although Form II homodimer has a similar contact surface (over $700 \AA^{2}$ ) and interactions between two molecules in one dimer, Form I homodimer, which provides about $810 \AA^{2}$ of intra-dimer interface, may have a higher possibility to be characterized as the functional homodimer, which is indicated by relevant research on the ANAC NAC domain (Ernst et al., 2004).
The interface between the two monomers primarily consists of residues in the long loop region formed by $\mathrm{N}$ terminal residues and another two residues in the $\alpha 1$ helix. These residues include Leu14 to Thr23, Glu26 and Tyr31, together with their counterparts in the second monomer (Fig. 1B), and form a short anti-parallel $\beta$-sheet at the dimer interface similar to the ANAC NAC domain. Arg68, which is located in the $\beta 1$ strand, has additional hydrogen bonds with His21 and Thr23 in the other monomer for inter-monomer interaction. Because of the high sequence similarity in this interface of SNAC1 and the ANAC NAC domain, two prominent salt bridges formed by the conserved Arg19 and Glu26 could also be observed in the structure of SNAC1 NAC domain. Although the accessible surface of interface is $812 \AA^{2}$ $(10 \%$ of total) for each monomer, the strong dimerization in solution indicates that the interaction between two monomers via hydrophobic ineraction, hydrogen bond, salt bridge and van de Waals interaction (Table 1) should be very strong and 
A



B

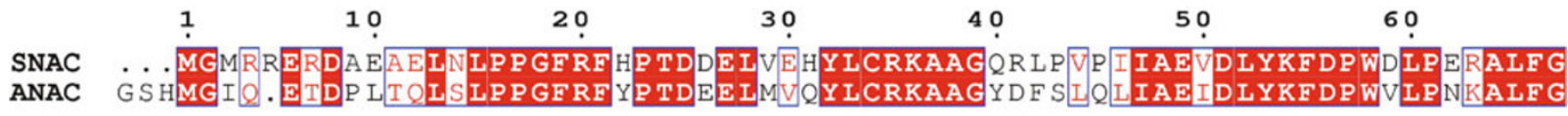



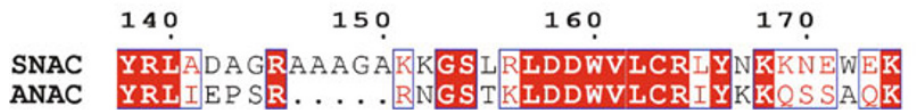

Figure 2. Comparison of SNAC1 and ANAC NAC domains. (A) Superposition of SNAC1 NAC domain (red ribbon) and ANAC NAC domain (yellow ribbon) is presented. (B) Sequence alignment of SNAC1 NAC domain and ANAC NAC domain.

Table 1 The dimer interface of SNAC1 NAC domain

\begin{tabular}{lc}
\hline Interface parameters & \\
\hline Accessible surface area $\left(\AA^{2}\right)$ & 812 \\
$\%$ of monomer accessible surface area & 9.2 \\
\% Polar residues in interface 25.00 & 18.18 \\
$\%$ Non-polar residues in interface & 55.00 \\
$\%$ Charged residues in interface & 20.00 \\
Hydrogen bonds & 12 \\
Salt bridges & 22 \\
\hline
\end{tabular}

suggests that the homodimer should be stable when the SNAC1 NAC domain exists in solution. However, and also suggested by the previously reported structure of ANAC NAC domain, more biologic evidence is needed to ascertain whether this crystallography related dimer has biologic relevance or not.

\section{A possible mechanism for DNA binding}

NAC transcription factors are related to a variety of plantspecific reactions, such as development of plant-specific organs, responses to plant hormones (Ernst et al., 2004), and 
A

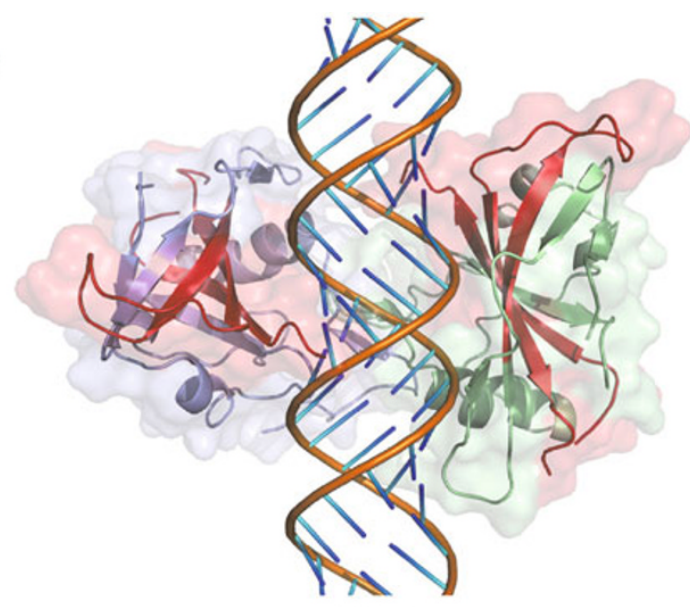

Top view

B

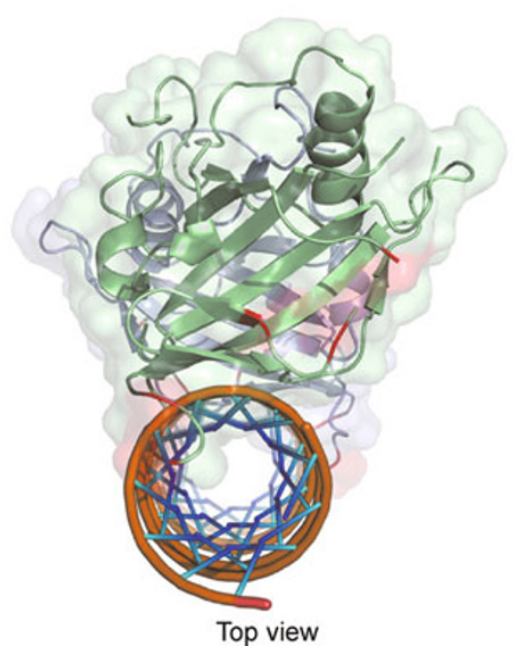

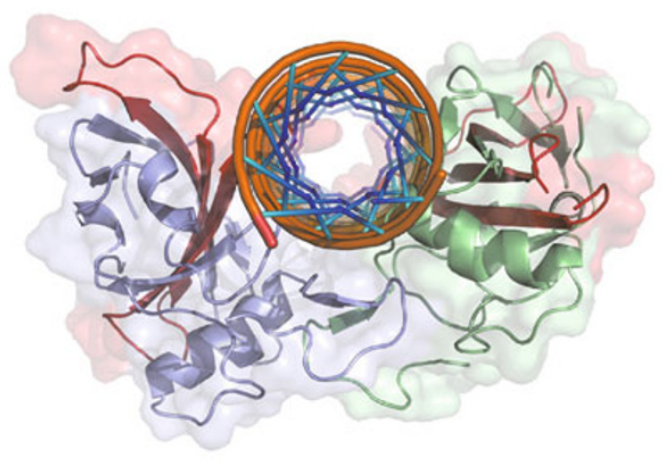

Side view

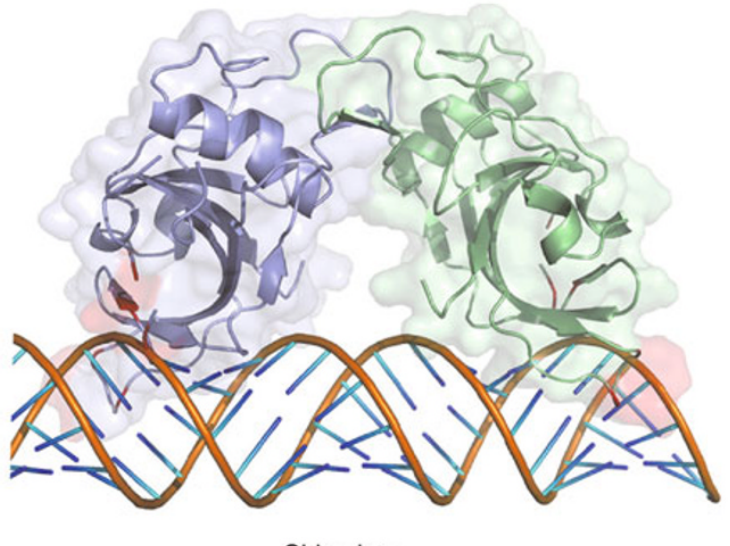

Side view

Figure 3. Structure details of the essential loop for SNAC1 NAC domain DNA binding. The SNAC1 NAC domain molecule is represented as while ribbon. Arg88 and Arg85 are shown as gold and cyan sticks; other residues, which might be crucial for loop stabilization and DNA binding, are shown as green sticks. The hydrogen bonds between Arg88 and interacting residues are shown as dashed lines.

responses to drought and high salinity stresses (Hu et al., 2006). Although there is still a lack of sufficient structural data to firmly establish precise understanding of the SNAC1 NAC domain binding with DNA, previously reported biochemical and crystallographic results allow us to build a possible model for SNAC1 NAC domain binding with DNA.

NAC transcription factors belong to the second largest family of the plant-specific transcription factors together with NAM, ATAF1,2, and CUC2 (cup-shaped cotyledon), sharing a conserved DNA binding domain of $\sim 150$ amino acids in length named as the NAC domain (Hu et al., 2006). WRKY proteins comprise another transcription factor family, which are essential in pathogen and salicylic acid responses of higher plants (Yamasaki et al., 2005). WRKY proteins share a highly conserved DNA binding domain known as the WRKY domain, which consists of a $\mathrm{CX}_{4-5} \mathrm{CX}_{22-23} \mathrm{HXH}$ zinc binding motif and a conserved WRKYGQK sequence (Yamasaki et al., 2005). The structure of the C-terminal WRKY domain of the Arabidopsis thaliana WRKY4 protein consists of a central four-stranded $\beta$-sheet, with a zinc binding pocket formed by conserved $\mathrm{Cys} / \mathrm{His}$ residues located at one end of the $\beta$ sheet. Further biochemical results suggest that this zinc finger should be responsible for WRKY DNA binding, through which the zinc finger and the conserved WRKYGQK motif enters the DNA groove and forms contacts with the DNA bases (Yamasaki et al., 2005). The result of Secondary Structure Matching (Krissinel and Henrick, 2004) indicates that the central four strands of the SNAC1 NAC domain shares a high structural similarity with the four-stranded $\beta$-sheet of WRKY domain. However, no zinc finger in the SNAC1 NAC domain is 
observed, which is consistent with atomic absorption spectroscopy and the reported structure of ANAC. Moreover, WRKY proteins will function in monomeric form according to current knowledge. All these results suggest that the SNAC1 NAC domain should have a distinct DNA binding mode with WRKY, though they share a high structural similarity in regards to their central structure.

Duval and colleagues first identified the DNA binding domain of AtANM between Val119 and Ser183 (AtANM numbering) in the central four stranded $\beta$-sheet by using yeast one hybrid assay (Duval et al., 2002). Except for the loop region between Ala141-Arg156 (SNAC1 NAC domain numbering), the high sequence similarity between AtANM, ANAC and SNAC1 NAC domain indicates a structural similarity among them. Therefore, it is conceivable that the central $\beta$-sheet of the SNAC1 NAC domain will play a key role in DNA binding (Fig. 3A). In a contrast, mutagenesis studies of ANAC showed that two areas of the ANAC NAC domain, which contain the positively charged residues Lys 123 and Lys126 (ANAC) positioned between $\beta 4$ and 5 on a turn protruding from the surface of the domain in addition to Lys79 and Arg85 (ANAC) positioned in a loop between $\beta 1$ and $\beta 2$, as well as Arg88, the first residue in $\beta 2$, take the responsibility of ANAC DNA binding. While K123A/K126A mutants did not impair the DNA binding of ANAC, both K79A/R85A/R88A triple mutants and R85A/R88A double mutants abolished the DNA binding (Olsen et al., 2005). As size-exclusion chromatography showed proper folding of all these mutant domains, Arg85, Arg88 or both are therefore biochemically important for binding. Although Arg85/Arg88 was missing in the structure of the ANAC NAC domain, the unambiguous electron density allows us to build them properly in the structure of SNAC1 NAC domain (Fig. 4). The side chain of Arg85 is exposed to the solution and thus may indicate that the side chain of Arg85

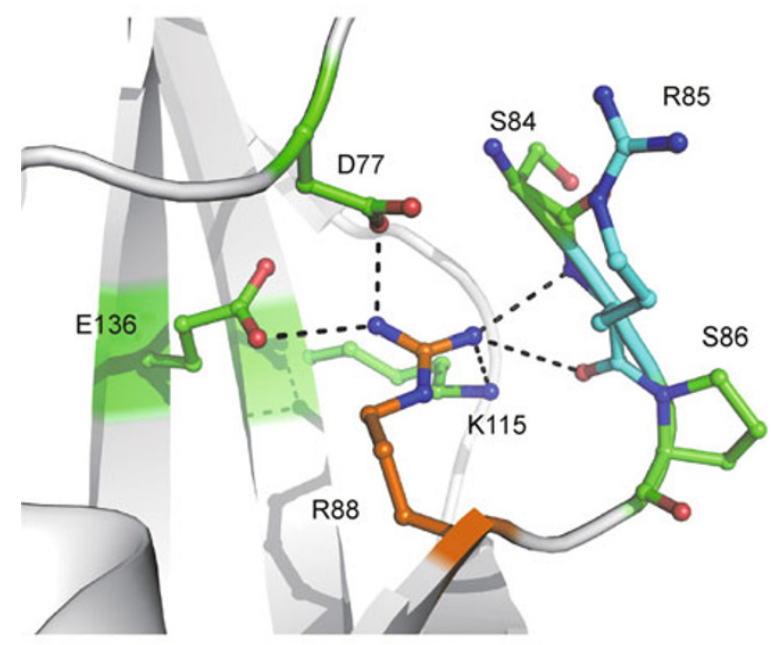

Figure 4. Proposed models of SNAC1 NAC domain binding with DNA according to the results reported by Duval and Olsen, respectively. will be involved in DNA binding. However, the side chain of Arg88 is buried in a pocket, which is mainly formed by Asp77, Lys115, Glu136 and main chain atoms of Ser84-Pro86, and thus well stabilized by a hydrogen bond network. Therefore, the side chain of Arg88 is not likely to be directly involved in DNA biding and we would like to propose that Arg88 takes the major responsibility to stabilize the loop region and thus affect the DNA binding of the SNAC1 NAC domain. However, although both of the reported biochemical results present clues for understanding the DNA binding of the SNAC1 NAC domain, inconsistencies between the reported results indicate that further experimental structural information is necessary to provide a precise model of NAC domain binding to DNA substrate.

\section{CONCLUSIONS}

SNAC1 (Stress-Responsive NAC 1) from rice belongs to the NAC protein family, which is crucial for plant development, transcription regulation and regulatory pathways involving protein-protein interactions. Furthermore NAC proteins significantly enhance drought resistance while showing no phenotypic changes or yield penalty. In this report, we report the crystal structure of the SNAC1 NAC domain. The structure of the SNAC1 NAC domain shares a similarity with a transcription factor fold identified by ANAC NAC domain, and consists of a central semi- $\beta$-barrel formed by seven twisted anti-parallel $\beta$-strands with three $\alpha$-helices on one side and the open side respectively. The structure also suggests that this domain mediates dimerization of the NAC proteins through conserved interactions and indicates that the dimerization should be conserved in NAC family. Moreover, stabilization of the loop region between $\beta 1$ and 2 by Arg88 is hypothesized to be responsibile for DNA binding.

\section{MATERIALS AND METHODS}

\section{Protein expression, purification and characterization}

The NAC domain of the SNAC1 protein coding sequence was amplified and inserted into the pET-28a plasmid (GE Healthcare) with $6 \times$ His tag at the $\mathrm{N}$ terminus using Ndel and Xhol sites (shown in bold). The forward and reverse PCR primers used for amplification were: forward (5'-CATATGGGGATGAGGAGGGAGAGAGG-3') and reverse (5'-CTCGAGTCACTTCTCCCACTCGTTCTTCTTGTTG-3'). The plasmid was transformed into E. coli strain BL21 (DE3), and cells were cultured at $310 \mathrm{~K}$ in LB media containing $100 \mathrm{mg} / \mathrm{L}$ ampicillin. After the $\mathrm{OD}_{600}$ reached 0.6 , the culture was cooled to $289 \mathrm{~K}$ and supplemented with $0.5 \mathrm{mM}$ IPTG. After an overnight induction, the cells were harvested by centrifugation at 5000 rotation/ $\min (10 \mathrm{~min}, 277 \mathrm{~K})$. The cell pellets were resuspended in lysis buffer ( $50 \mathrm{mM}$ Tris- $\mathrm{HCl} \mathrm{pH} 8.0,500 \mathrm{mM} \mathrm{NaCl}, 20 \mathrm{mM}$ imidazole, $10 \%$ glycerol) and disrupted by high pressure. The cell debris was removed by centrifugation at 12,000 rotation/min ( $30 \mathrm{~min}, 277 \mathrm{~K}$ ). The His-tagged target protein was purified by Ni-NTA affinity chromatography and eluted by lysis buffer with an additional $250 \mathrm{mM}$ imidazole. The eluted protein was further purified by gel 
filtration with buffer containing $25 \mathrm{mM}$ HEPES pH 7.5 and $150 \mathrm{mM}$ $\mathrm{NaCl}$. The target protein was examined to have a purity over $95 \%$ by SDS-PAGE and concentrated to $20 \mathrm{mg} / \mathrm{mL}$ before storage at $-80^{\circ} \mathrm{C}$.

\section{Crystallization}

Crystallization was performed at $290 \mathrm{~K}$ by the hanging-drop vapor diffusion technique. Crystals were obtained by mixing $1 \mu \mathrm{L}$ of protein solution with an equal volume of reservoir solution. The initial crystals appeared after three months with ammonium sulfate as the precipitation reagent. The rod shaped crystals with good diffraction quality grew to a final size of $100 \mu \mathrm{m} \times 100 \mu \mathrm{m} \times 200 \mu \mathrm{m}$ in the optimized crystallization buffer containing $0.1 \mathrm{M} \mathrm{HEPES} \mathrm{pH} 7.5$ and $1.5 \mathrm{M}$ ammonium sulfate. The crystals were then soaked in a cryoprotectant solution consisting of the reservoir solution and $20 \%(\mathrm{~V} / \mathrm{V})$ glycol and then flash-frozen in liquid nitrogen for X-ray diffraction data collection. The crystals belong to space group $P 4_{1}{ }_{2} 2$, with cell parameters $\alpha=b=45.9 \AA, c=176.5 \AA, \alpha=\beta=\gamma=90^{\circ}$.

\section{X-ray data collection, processing and structure determination}

The crystal of the NAC domain of SNAC1 diffracted to $2.5 \AA$ resolution at $100 \mathrm{~K}$ in BL17A (Photon Facoty, Japan) with an ADSC Q315 CCD detector at the wavelength of $1.0000 \AA$. Data were processed, integrated, and scaled using the HKL2000 package (Otwinowski and Minor, 1997). The crystals belong to the $P 4_{1} 2{ }_{1} 2$ space group with unit cell parameters of $\alpha=b=45.9 \AA, c=176.5 \AA, \alpha=\beta=\gamma=90^{\circ}$. There is only one molecule per asymmetric unit with a Matthews coefficient of $2.6 \AA^{3} / \mathrm{Da}$, corresponding to $43 \%$ solvent content (Collaborative Computational Project, 1994).

The PHASER program (McCoy et al., 2007) was used to find the correct solution through the molecular replacement method by using the crystal structure of NAC domain of ANAC (PDB code: 1UT4) (Ernst et al., 2004) as the initial searching model. Clear solution with a high rotation Z-socre (7.4) and translation Z-score (10.3) indicates a correct searching solution. Manual model building and refinement were performed with COOT (Emsley and Cowtan, 2004) and PHENIX (Adams et al., 2002). During the later stages of positional refinement, restraints were relaxed, and a bulk solvent correction was applied under the guidance of $R_{\text {free }}$. Model geometry was verified using the program PROCHECK (Laskowski et al., 1993). Solvent molecules were located from stereochemically reasonable peaks in the $\sigma A$ weighted Fo-Fc difference electron density map. Final refinement statistics are summarized in Table 2. Structural figures were drawn with the program PyMOL (DeLano, 2002).

\section{ACKNOWLEDGEMENTS}

We thank staffs from BSRF and Photon Factory for their technical assistance with data collection. This work was supported by the

Table 2 Data collection and refinement statistics

\begin{tabular}{|c|c|}
\hline Data collection statistics & \\
\hline Cell parameters $\left(\AA,{ }^{\circ}\right)$ & $\alpha=b=45.97 \AA, c=176.5 \AA, \alpha=\beta=\gamma=90^{\circ}$ \\
\hline Space group & $P 4{ }_{1}{ }_{1} 2$ \\
\hline Wavelength used $(\AA)$ & 1.0000 \\
\hline Resolution $(\AA)$ & $50.0(2.6)^{\mathrm{c}}-2.5$ \\
\hline No. of all reflections & 129,792 \\
\hline No. of unique reflections & 7014 \\
\hline Completeness (\%) & $99.7(96.8)$ \\
\hline Average $\mathrm{I} / \sigma(\mathrm{I})$ & $11.5(3.2)$ \\
\hline$R_{\text {merge }}^{\text {a }}(\%)$ & $6.4(50.9)$ \\
\hline \multicolumn{2}{|l|}{ Refinement statistics } \\
\hline No. of reflections used $(\sigma(F)>0)$ & 6658 \\
\hline$R_{\text {work }}{ }^{\mathrm{b}}(\%)$ & 23.4 \\
\hline$R_{\text {free }}{ }^{\mathrm{b}}(\%)$ & 27.1 \\
\hline r.m.s.d. bond distance $(\AA)$ & 0.010 \\
\hline r.m.s.d. bond angle $\left({ }^{\circ}\right)$ & 1.514 \\
\hline Average $B$-value $\left(\AA^{2}\right)$ & 62.3 \\
\hline \multicolumn{2}{|c|}{ Ramachandran plot (excluding Pro and Gly) } \\
\hline Res. in most favored regions & $92(77.3 \%)$ \\
\hline Res. in additionally allowed regions & $27(22.7 \%)$ \\
\hline Res. in generously allowed regions & $0(0.0 \%)$ \\
\hline
\end{tabular}


National Natural Science Foundation of China (Grant Nos. 30730022 and 30870486), the National Basic Research Program (973 Program) (Grant No. 2007CB914304), and the National Major Projects (grant Nos. 2009ZX09311-001 and 2009ZX10004-304).

\section{REFERENCES}

Adams, P.D., Grosse-Kunstleve, R.W., Hung, L.W., loerger, T.R., McCoy, A.J., Moriarty, N.W., Read, R.J., Sacchettini, J.C., Sauter, N.K., and Terwilliger, T.C. (2002). PHENIX: building new software for automated crystallographic structure determination. Acta Crystallogr D Biol Crystallogr 58, 1948-1954.

Aida, M., Ishida, T., Fukaki, H., Fujisawa, H., and Tasaka, M. (1997). Genes involved in organ separation in Arabidopsis: an analysis of the cup-shaped cotyledon mutant. Plant Cell 9, 841-857.

Collaborative Computational Project, Number 4. (1994). The CCP4 suite: programs for protein crystallography. Acta Crystallogr D Biol Crystallogr 50, 760-763.

Collinge, M., and Boller, T. (2001). Differential induction of two potato genes, Stprx2 and StNAC, in response to infection by Phytophthora infestans and to wounding. Plant Mol Biol 46, 521-529.

DeLano, W.L. (2002). The PyMOL Molecular Graphics System. San Carlos, CA, USA: DeLano Scientific. http://www.pymol.org.

Delessert, C., Kazan, K., Wilson, I.W., Van Der Straeten, D., Manners, J., Dennis, E.S., and Dolferus, R. (2005). The transcription factor ATAF2 represses the expression of pathogenesis-related genes in Arabidopsis. Plant J 43, 745-757.

Demura, T., and Fukuda, H. (2007). Transcriptional regulation in wood formation. Trends Plant Sci 12, 64-70.

Du, J., and Groover, A. (2010). Transcriptional regulation of secondary growth and wood formation. J Integr Plant Biol 52, 17-27.

Duval, M., Hsieh, T.F., Kim, S.Y., and Thomas, T.L. (2002). Molecular characterization of AtNAM: a member of the Arabidopsis NAC domain superfamily. Plant Mol Biol 50, 237-248.

Emsley, P., and Cowtan, K. (2004). Coot: model-building tools for molecular graphics. Acta Crystallogr D Biol Crystallogr 60, 2126-2132.

Ernst, H.A., Olsen, A.N., Larsen, S., and Lo Leggio, L. (2004). Structure of the conserved domain of ANAC, a member of the NAC family of transcription factors. EMBO Rep 5, 297-303.

Fang, Y., You, J., Xie, K., Xie, W., and Xiong, L. (2008). Systematic sequence analysis and identification of tissue-specific or stressresponsive genes of NAC transcription factor family in rice. Mol Genet Genomics 280, 547-563.

Hegedus, D., Yu, M., Baldwin, D., Gruber, M., Sharpe, A., Parkin, I., Whitwill, S., and Lydiate, D. (2003). Molecular characterization of Brassica napus NAC domain transcriptional activators induced in response to biotic and abiotic stress. Plant Mol Biol 53, 383-397.

Hu, H., Dai, M., Yao, J., Xiao, B., Li, X., Zhang, Q., and Xiong, L. (2006). Overexpressing a NAM, ATAF, and CUC (NAC) transcription factor enhances drought resistance and salt tolerance in rice. Proc Natl Acad Sci U S A 103, 12987-12992.

Hu, R., Qi, G., Kong, Y., Kong, D., Gao, Q., and Zhou, G. (2010). Comprehensive analysis of NAC domain transcription factor gene family in Populus trichocarpa. BMC Plant Biol 10, 145-167.

Jensen, M.K., Hagedorn, P.H., de Torres-Zabala, M., Grant, M.R., Rung, J.H., Collinge, D.B., and Lyngkjaer, M.F. (2008). Transcrip- tional regulation by an NAC (NAM-ATAF1,2-CUC2) transcription factor attenuates $A B A$ signalling for efficient basal defence towards Blumeria graminis f. sp. hordei in Arabidopsis. Plant J 56, 867-880. Jensen, M.K., Rung, J.H., Gregersen, P.L., Gjetting, T., Fuglsang, A. T., Hansen, M., Joehnk, N., Lyngkjaer, M.F., and Collinge, D.B. (2007). The HvNAC6 transcription factor: a positive regulator of penetration resistance in barley and Arabidopsis. Plant Mol Biol 65, 137-150.

Kim, S.G., Kim, S.Y., and Park, C.M. (2007). A membrane-associated NAC transcription factor regulates salt-responsive flowering via FLOWERING LOCUS T in Arabidopsis. 0032-0935 226, 64-654.

Kim, Y.S., Kim, S.G., Park, J.E., Park, H.Y., Lim, M.H., Chua, N.H., and Park, C.M. (2006). A membrane-bound NAC transcription factor regulates cell division in Arabidopsis. Plant Cell 18, 3132-3144.

Ko, J.H., Yang, S.H., Park, A.H., Lerouxel, O., and Han, K.H. (2007). ANAC012, a member of the plant-specific NAC transcription factor family, negatively regulates xylary fiber development in Arabidopsis thaliana. Plant J 50, 1035-1048.

Krissinel, E., and Henrick, K. (2004). Secondary-structure matching (SSM), a new tool for fast protein structure alignment in three dimensions. Acta Crystallogr D Biol Crystallogr 60, 2256-2268.

Kubo, M., Udagawa, M., Nishikubo, N., Horiguchi, G., Yamaguchi, M., Ito, J., Mimura, T., Fukuda, H., and Demura, T. (2005). Transcription switches for protoxylem and metaxylem vessel formation. Genes Dev 19, 1855-1860.

Laskowski, R., MacArthur, M., Moss, D., and Thornton, J. (1993). PROCHECK: a program to check the stereochemical quality of protein structures. J Appl Cryst 26, 283-291.

Lu, P.-L., Chen, N.-Z., An, R., Su, Z., Qi, B.-S., Ren, F., Chen, J., and Wang, X.-C. (2007). A novel drought-inducible gene, ATAF1, encodes a NAC family protein that negatively regulates the expression of stress-responsive genes in \&lt;i\&gt;Arabidopsis\&lt;/ i\&gt. 0167-4412 63, 289-305.

Matthews, B.W. (1968). Solvent content of protein crystals. J Mol Biol 33, 491- 497.

McCarthy, R.L., Zhong, R., and Ye, Z.H. (2009). MYB83 is a direct target of SND1 and acts redundantly with MYB46 in the regulation of secondary cell wall biosynthesis in Arabidopsis. Plant Cell Physiol 50, 1950-1964.

McCoy, A.J., Grosse-Kunstleve, R.W., Adams, P.D., Winn, M.D., Storoni, L.C., and Read, R.J. (2007). Phaser crystallographic software. J Appl Crystallogr 40, 658-674.

Mitsuda, N., Iwase, A., Yamamoto, H., Yoshida, M., Seki, M., Shinozaki, K., and Ohme-Takagi, M. (2007). NAC transcription factors, NST1 and NST3, are key regulators of the formation of secondary walls in woody tissues of Arabidopsis. Plant Cell 19, 270-280.

Müller, C.W. (2001). Transcription factors: global and detailed views. Curr Opin Struct Biol 11, 26-32.

Nakashima, K., Tran, L.S., Van Nguyen, D., Fujita, M., Maruyama, K., Todaka, D., Ito, Y., Hayashi, N., Shinozaki, K., and YamaguchiShinozaki, K. (2007). Functional analysis of a NAC-type transcription factor OsNAC6 involved in abiotic and biotic stress-responsive gene expression in rice. Plant J 51, 617-630.

Ohnishi, T., Sugahara, S., Yamada, T., Kikuchi, K., Yoshiba, Y., Hirano, H.Y., and Tsutsumi, N. (2005). OsNAC6, a member of the NAC gene family, is induced by various stresses in rice. Genes 
Genet Syst 80, 135-139.

Olsen, A.N., Ernst, H.A., Leggio, L.L., and Skriver, K. (2005). DNAbinding specificity and molecular functions of NAC transcription factors. 0168-9452 169, 785-797.

Ooka, H., Satoh, K., Doi, K., Nagata, T., Otomo, Y., Murakami, K., Matsubara, K., Osato, N., Kawai, J., Carninci, P., et al. (2003). Comprehensive analysis of NAC family genes in Oryza sativa and Arabidopsis thaliana. DNA Res 10, 239-247.

Otwinowski, Z., and Minor, W. (1997). Processing of X-ray diffraction data collected in oscillation mode. In: Macromolecular Crystallography, part A. C.W. Carter Jr., and R.M. Sweet, eds. San Diego, CA: Academic Press. 307-326.

Riechmann, J.L., Heard, J., Martin, G., Reuber, L., Jiang, C., Keddie, J., Adam, L., Pineda, O., Ratcliffe, O.J., Samaha, R.R., et al. (2000). Arabidopsis transcription factors: genome-wide comparative analysis among eukaryotes. Science 290, 2105-2110.

Sablowski, R.W.M., and Meyerowitz, E.M. (1998). A homolog of NO APICAL MERISTEM is an immediate target of the floral homeotic genes APETALA3/PISTILLATA. Cell 92, 93-103.

Souer, E., van Houwelingen, A., Kloos, D., Mol, J., and Koes, R. (1996). The no apical meristem gene of Petunia is required for pattern formation in embryos and flowers and is expressed at meristem and primordia boundaries. Cell 85, 159-170.

Sperotto, R.A., Ricachenevsky, F.K., Duarte, G.L., Boff, T., Lopes, K. L., Sperb, E.R., Grusak, M.A., and Fett, J.P. (2009). Identification of up-regulated genes in flag leaves during rice grain filling and characterization of OsNAC5, a new ABA-dependent transcription factor. 0032-0935 230, 985-1002.

Takasaki, H., Maruyama, K., Kidokoro, S., Ito, Y., Fujita, Y., Shinozaki, K., Yamaguchi-Shinozaki, K., and Nakashima, K. (2010). The abiotic stress-responsive NAC-type transcription factor OsNAC5 regulates stress-inducible genes and stress tolerance in rice. Mol Genet Genomics 284, 173-183.

Tran, L.S., Nakashima, K., Sakuma, Y., Simpson, S.D., Fujita, Y., Maruyama, K., Fujita, M., Seki, M., Shinozaki, K., and YamaguchiShinozaki, K. (2004). Isolation and functional analysis of Arabidopsis stress-inducible NAC transcription factors that bind to a drought-responsive cis-element in the early responsive to dehydration stress 1 promoter. Plant Cell 16, 2481-2498.

Uauy, C., Distelfeld, A., Fahima, T., Blechl, A., and Dubcovsky, J. (2006). A NAC Gene regulating senescence improves grain protein, zinc, and iron content in wheat. Science 314, 1298-1301.

Wang, X.e., Basnayake, B.M.V.S., Zhang, H., Li, G., Li, W., Virk, N., Mengiste, T., and Song, F. (2009). The Arabidopsis ATAF1, a NAC Transcription Factor, Is a Negative Regulator of Defense Responses Against Necrotrophic Fungal and Bacterial Pathogens. Molecular Plant-Microbe Interactions 22, 1227-1238.

Weir, I., Lu, J., Cook, H., Causier, B., Schwarz-Sommer, Z., and Davies, B. (2004). CUPULIFORMIS establishes lateral organ boundaries in Antirrhinum. 0950-1991 131, 915-922.

Wu, Y., Deng, Z., Lai, J., Zhang, Y., Yang, C., Yin, B., Zhao, Q., Zhang, L., Li, Y., Yang, C., et al. (2009). Dual function of Arabidopsis ATAF1 in abiotic and biotic stress responses. Cell Res 19, 1279-1290.

Xie, Q., Frugis, G., Colgan, D., and Chua, N.H. (2000). Arabidopsis NAC1 transduces auxin signal downstream of TIR1 to promote lateral root development. Genes Dev 14, 3024-3036.

Xiong, Y., Liu, T., Tian, C., Sun, S., Li, J., and Chen, M. (2005). Transcription factors in rice: a genome-wide comparative analysis between monocots and eudicots. Plant Mol Biol 59, 191-203.

Yamasaki, K., Kigawa, T., Inoue, M., Tateno, M., Yamasaki, T., Yabuki, T., Aoki, M., Seki, E., Matsuda, T., Tomo, Y., et al. (2005). Solution structure of an Arabidopsis WRKY DNA binding domain. Plant Cell 17, 944-956.

Zhong, R., Demura, T., and Ye, Z.H. (2006). SND1, a NAC domain transcription factor, is a key regulator of secondary wall synthesis in fibers of Arabidopsis. Plant Cell 18, 3158-3170.

Zhong, R., Richardson, E.A., and Ye, Z.H. (2007a). The MYB46 transcription factor is a direct target of SND1 and regulates secondary wall biosynthesis in Arabidopsis. Plant Cell 19, 2776-2792.

Zhong, R., Richardson, E.A., and Ye, Z.H. (2007b). Two NAC domain transcription factors, SND1 and NST1, function redundantly in regulation of secondary wall synthesis in fibers of Arabidopsis. Planta 225, 1603-1611. 